\title{
"First time right" - calculating imaging systems from scratch - INVITED
}

\author{
Fabian Duerr ${ }^{l, *}$, and Hugo Thienpont ${ }^{1}$ \\ ${ }^{1}$ Brussels Photonics, Department of Applied Physics and Photonics, Vrije Universiteit Brussel, Pleinlaan 2, 1050, Brussels, Belgium
}

\begin{abstract}
Freeform optics can be used to greatly extend the functionalities, improve performance, and reduce the volume and weight of optical systems. Today, the design of imaging systems largely relies on efficient ray tracing and optimization algorithms. Such a "step-and-repeat" approach to optical design typically requires considerable experience, intuition, and eventually "trial-and-error" guesswork. This time-consuming process applies especially to freeform optical systems. In this work, we present a deterministic direct optical design method for freeform imaging systems based on differential equations derived from Fermat's principle and solved using power series. The method allows calculating all optical surface coefficients that ensure minimal image blurring for each individual order of aberrations. We demonstrate the systematic, deterministic, scalable, and holistic character of our method with several catoptric and catadioptric design examples.
\end{abstract}

\section{Introduction}

For more than 150 years, scientists have advanced aberration theory to describe, analyse and eliminate imperfections that disturb the imaging quality of optical components and systems. Simultaneously, they have developed design methods and manufacturing techniques for imaging systems with ever-increasing complexity and performance up to the point where they are now including optical elements that are unrestricted in their surface shape. These so-called freeform elements offer degrees of freedom that can greatly extend the functionalities and further boost the specifications of state-of-the-art imaging systems. However, the drastically increased number of surface coefficients of these freeform surfaces poses considerable challenges for the optical design process.

Today the design of optical systems largely relies on efficient raytracing and optimization algorithms for which a variety of (commercial) software and optimization algorithms are available. During an optical design cycle, different parameters and variables of the optical system are varied to optimize a defined merit function that indicates the image quality for a given field of view. These merit functions are typically "wild" with many local minima, and there is no guarantee that local or global optimization algorithms will lead to a satisfactory design solution. A successful and widely used optimizationbased optical design strategy therefore consists of choosing a well-known optical system as a starting point (e.g. from literature) and steadily achieving incremental improvements. However, this "step-and-repeat" approach to optical design does require considerable experience, intuition, and "trial-and-error" guesswork. This applies especially to freeform optical systems.
In this work, we review the recently presented "first time right" optical design method for freeform imaging systems. It is based on solving differential equations derived from Fermat's principle of least time by using a power series approach. The method allows calculating all optical surface coefficients that ensure minimal image blurring for each individual order of aberrations. As such, it offers a disruptive methodology to design optical imaging systems from scratch to facilitate a fast-track uptake of freeform elements in the next generation of high-end imaging systems. Further mathematical details can be found in a recently published article [1].

\section{Mathematical formulation}

There are numerous possibilities to describe aberrations in freeform optical systems according to their symmetry: (1) two orthogonal planes of symmetry; (2) one plane of symmetry; (3) without any symmetry. Here, we will focus on systems with one plane of symmetry as it is the most common category. Following an ansatz similar to that used in most aberration theories, we assume an arbitrary ray that is emitted from an object at infinity with a field direction vector $\mathbf{H}$ for field angles $\mathrm{H}_{\mathrm{x}}$ and $\mathrm{H}_{\mathrm{y}}$ that passes through the pupil of the system (e.g., first surface in Fig. 1) at $\left(x_{p}, y_{p}, f_{1}\left(x_{p}, y_{p}\right)\right)$, and that finally intersects the image plane at $\mathbf{h}=\left(\mathrm{h}_{\mathrm{x}}, \mathrm{h}_{\mathrm{y}}, \mathrm{D}\right)$. This intersection can be written in vector form as a series expansion $\mathbf{h}=\mathbf{h}_{0}+\epsilon^{(1)}+\epsilon^{(2)}+\epsilon^{(3)}$ $+\ldots$ where $\mathbf{h}_{0}=\left(\mathrm{g}\left(\mathrm{H}_{\mathrm{x}}\right), \mathrm{g}\left(\mathrm{H}_{\mathrm{y}}\right), \mathrm{D}\right)$ denotes the ideal (aberration-free) image location as a function of $\mathrm{H}_{\mathrm{x}}$ and $\mathrm{H}_{\mathrm{y}}$. Deviations from the ideal image location are then described by ray aberrations polynomials $\epsilon^{(i)}=\left(\epsilon_{x}, \epsilon_{y}, 0\right)$ with the transverse ray aberration series $\epsilon_{\mathrm{x}}\left(\mathrm{x}_{\mathrm{p}}, \mathrm{y}_{\mathrm{p}}, \mathrm{H}_{\mathrm{x}}, \mathrm{H}_{\mathrm{y}}\right)$ and $\epsilon_{\mathrm{y}}\left(\mathrm{x}_{\mathrm{p}}, \mathrm{y}_{\mathrm{p}}, \mathrm{H}_{\mathrm{x}}, \mathrm{H}_{\mathrm{y}}\right)$, in $\mathrm{x}$ - and $\mathrm{y}$-direction, respectively.

\footnotetext{
* Corresponding author: fduerr@b-phot.org
} 


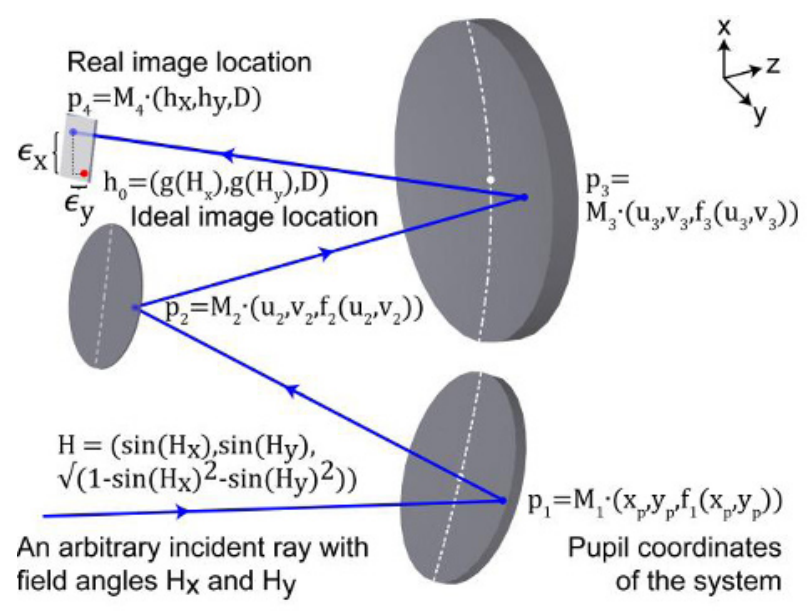

Fig. 1. Schematic layout of a typical plane-symmetric freeform optical system. The ray path of an arbitrary ray is shown including the introduced mathematical description of the initial ray direction and intersections with each mirror and the local transverse ray aberrations $\epsilon_{\mathrm{x}}$ and $\epsilon_{\mathrm{y}}$ in the image plane.

We now consider a sequence of $\mathrm{N}$ refractive and/or reflective optical surfaces $f_{i}(i=1 \ldots N)$ (in Fig. 1 these are three mirrors) aligned along a principal ray path. To describe their surface functions, we use a XY-polynomial representation $f_{i}(x, y)$ with series coefficients $f_{i, s t}$. Next, we introduce ray mapping functions $\left(\mathrm{u}_{\mathrm{i}}, \mathrm{v}_{\mathrm{i}}\right)$ in $\mathrm{x}$ - and $\mathrm{y}$ direction that describe where an arbitrary ray, described by variables $\left(\mathrm{x}_{\mathrm{p}}, \mathrm{y}_{\mathrm{p}}, \mathrm{H}_{\mathrm{x}}, \mathrm{H}_{\mathrm{y}}\right)$, will intersect each optical surface. For individual surface orientations, we define the tilts of all elements through rotation matrices $\mathbf{M}_{\mathbf{i}}$. The ray path from the object to the image plane then consists of $(\mathrm{N}+1)$ segments $\mathrm{d}_{1} \ldots \mathrm{d}_{\mathrm{N}+1}$ that describe the optical path length distances for an arbitrary ray. Vector geometry enables us to express these distances weighted by the refractive indices $n_{i, i+1}$ of the surrounding materials. By applying Fermat's principle, we can derive two sets of differential equations for all defined distances $d_{i}$ pairwise from object to image space

$$
\begin{array}{ll}
\mathrm{D}_{i, x}=\partial_{\mathrm{u}_{\mathrm{i}}}\left(\mathrm{d}_{\mathrm{i}}+\mathrm{d}_{\mathrm{i}+1}\right)=0 & (\mathrm{i}=1 \ldots \mathrm{N}) \\
\mathrm{D}_{i, y}=\partial_{\mathrm{v}_{\mathrm{i}}}\left(\mathrm{d}_{\mathrm{i}}+\mathrm{d}_{\mathrm{i}+1}\right)=0 & (\mathrm{i}=1 \ldots \mathrm{N})
\end{array}
$$

An optical system consisting of $\mathrm{N}$ surfaces is thus fully described by $N$ differential equations $D_{i, x}$ and $N$ differential equations $D_{i, y}$ for $i=1 \ldots N$, for a given but arbitrary pupil plane.

Suppose that the functions $h_{x}, h_{y}, u_{i}, v_{i}$ and $f_{i}$ are analytic and smooth solutions of the differential equations $\mathrm{D}_{\mathrm{i}, \mathrm{x}}$ and $\mathrm{D}_{\mathrm{i}, \mathrm{y}}$, then Taylor's theorem implies that the functions must be infinitely differentiable and have power series representations. We can employ a power series approach to seek solutions to the derived differential equations. This method substitutes the power series into the differential equations to determine the values of the series coefficients. To calculate the coefficients, we differentiate Eqs. (1) and (2) with respect to $\left(\mathrm{x}_{\mathrm{p}}, \mathrm{y}_{\mathrm{p}}, \mathrm{H}_{\mathrm{x}}, \mathrm{H}_{\mathrm{y}}\right)$, evaluated at $\mathrm{x}_{\mathrm{p}}=\mathrm{y}_{\mathrm{p}}=\mathrm{H}_{\mathrm{x}}=\mathrm{H}_{\mathrm{y}}=0$, such that

$$
\begin{array}{r}
\lim _{\mathrm{x}_{\mathrm{p}}, \mathrm{y}_{\mathrm{p}}, \mathrm{H}_{\mathrm{x}}, \mathrm{H}_{\mathrm{y}} \rightarrow 0} \frac{\partial^{j}}{\partial x_{p}^{j}} \frac{\partial^{k}}{\partial y_{p}^{k}} \frac{\partial^{l}}{\partial H_{x}^{l}} \frac{\partial^{m}}{\partial H_{y}^{m}} D_{i, x}=0,(i=1 . . N) \\
\lim _{\mathrm{x}_{\mathrm{p}}, \mathrm{y}_{\mathrm{p}}, \mathrm{H}_{\mathrm{x}}, \mathrm{H}_{\mathrm{y}} \rightarrow 0} \frac{\partial^{j}}{\partial x_{p}^{j}} \frac{\partial^{k}}{\partial y_{p}^{k}} \frac{\partial^{l}}{\partial H_{x}^{l}} \frac{\partial^{m}}{\partial H_{y}^{m}} D_{i, y}=0,(i=1 . . N)
\end{array}
$$

Once all system specifications such as the entrance pupil diameter, focal length, field of view and design wavelength are specified, we can establish the differential equations and initiate the method as follows: (1) solve the non-linear first order case $(\mathrm{j}+\mathrm{k}+\mathrm{l}+\mathrm{m}=1)$ using a standard non-linear solver; (2) solve the linear systems of equations in ascending order $(\mathrm{j}+\mathrm{k}+\mathrm{l}+\mathrm{m}=2,3, \ldots)$ by setting unwanted aberrations to zero or by minimizing a combination thereof as required by the targeted specifications of the imaging system. It is important to highlight that these two steps are virtually identical for all freeform designs. We calculate the surface coefficients up to the sixth order, and the mapping and aberration coefficients up to the eighth order throughout this work.

\section{Advanced freeform design examples}

The first design example is a catoptric imaging system proposed by Bauer et al. for the visible spectrum with $\mathrm{x}$ z-plane symmetry and the object at infinity [2]. It consists of three freeform mirrors with the stop at the first mirror, and a targeted system volume of 60 liters. Thus, the layout of the optical components can be described by three distances and four rotation angles (one for each mirror, and one for the image), which provides seven geometrical degrees of freedom for the optical designer. These distances and angles can now be (manually) adjusted to create an unobscured starting geometry that meets certain geometrical constraints such as the target volume. The directly calculated design that we obtained by only optimizing the seven degrees of freedom of the geometry is shown in Fig. 2a,

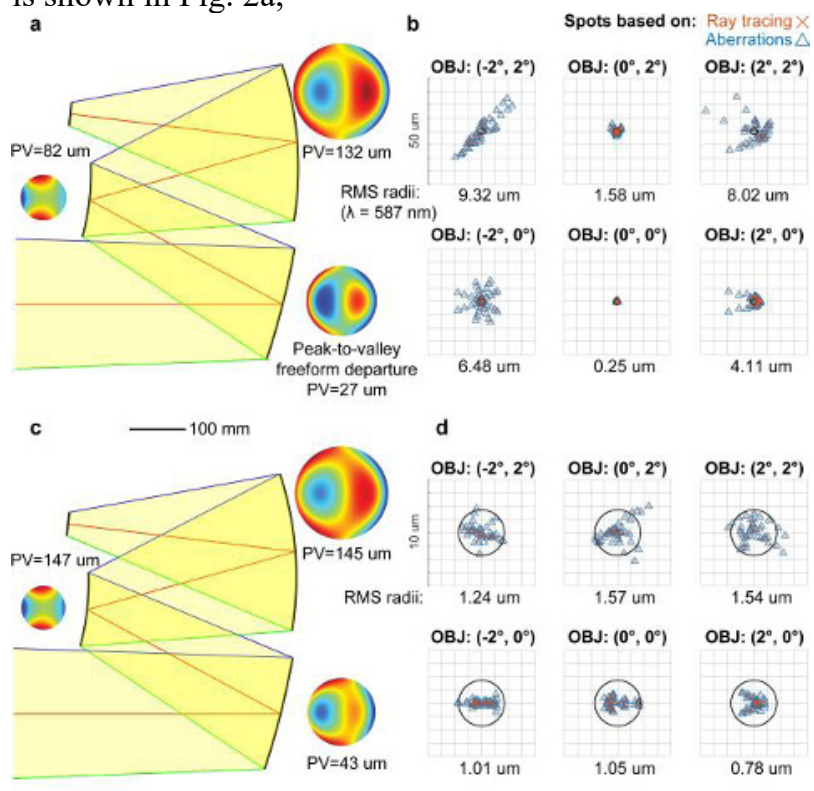

Fig. 2. "First time right" results for the three-mirror imager: a Cross-section of the directly calculated system. b Spot diagram for six fields based on aberration calculations and ray tracing in comparison. c Cross-section of the further optimized system. d Spot diagram for the identical six fields. 
where the system layout cross-section is combined with the full 3D peak-to-valley freeform departures (PV) from the best-fit base sphere for each mirror, respectively. Fig. $2 \mathrm{~b}$ shows the spot diagrams for six selected fields based on aberration calculations. With an average RMS spot radius of about 5 micrometers, our directly calculated system provides an already well-corrected "first time right" solution that can be readily further optimized. Thus, all forty previously calculated surface coefficients plus the initial seven degrees of freedom are used as variables for optimization, e.g. using MATLAB's lsqnonlin solver. After ten iterations and few minutes, the system already reaches diffraction-limited performance for almost the full field of view. The optimized design shows slightly increased and moderate freeform departures distributed among the three mirrors. The results are shown in Fig. 2c and $2 \mathrm{~d}$ accordingly. The aberration-based performance estimation of our method was found to be in excellent agreement with spot diagram data from classical ray tracing (calculated using Zemax, overlaid red cross symbols in Fig. 2b and 2d).

A second example is a monolithic freeform objective for a very compact infrared camera with four optical surfaces [3]. We chose this example to illustrate the scalability of our design method, well beyond the capabilities of most present-day direct freeform design approaches. We furthermore highlight its holistic character, since in this case we are dealing with a catadioptric system with freeform and aspherical surfaces. Here, the objective consists of three aspheres and one freeform at the second surface, with the stop placed at the first surface. We follow this reference as closely as possible: an F/1.4 design covering a $37 \times 25$-degree fieldof-view, an $8.4 \mathrm{~mm}$ entrance aperture, made of opticalgrade germanium and operating in the long-wave infrared region (LWIR) from 8-12 um. The layout of the system can be described by four distances and five angles, defining the principal ray path from object to image and the respective positions and orientation of all surfaces and corresponding rotation matrices. Optimizing the 9 initial degrees of freedom of the geometry yields an already well-corrected system. Fig. 3a shows the configuration of the system of comparable size to the cited reference design. The peak-to-valley freeform departure (PV) from the best-fit base sphere for the second freeform surface is added next to it. Fig. $3 \mathrm{~b}$ shows the corresponding spot diagrams for six selected fields (we used 15 fields for the optimization) based on our aberration calculations. With an average RMS spot radius of about 51 micrometers, we achieved an excellent starting point for further optimization. All surface coefficients are now set as variables for further optimization using MATLAB's lsqnonlin solver. The results are shown in Fig. 3c and 3d. Within few minutes, the system reaches a close to diffraction-limited performance in the LWIR band for the full field of view with an acceptable distortion of less than $5 \%$. Chromatic aberrations are very well controlled due to the relatively low dispersion of Germanium in the LWIR band. This spot diagram-based performance is in excellent agreement with spot diagram data from classical ray tracing.

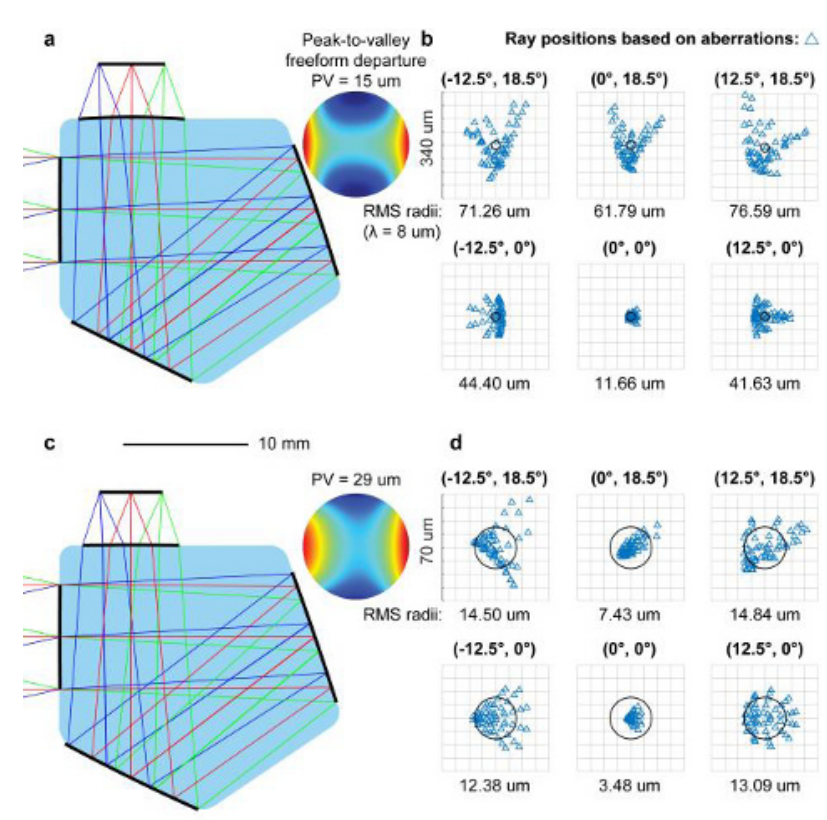

Fig. 3. "First time right" results for the monolithic LWIR design: a Cross-section of directly calculated system. b Spot diagram for six fields based on aberration calculations. $\mathbf{c}$ Cross-section of the further optimized system. d Spot diagram for identical six fields.

This catadioptric example clearly demonstrates yet again the highly effective nature of our proposed design and evaluation method. In addition, it highlights two further important features: (1) our method allows to combine all types of refractive and/or reflective surfaces of spherical, aspheric or freeform shapes; (2) the method enables to increase the number of calculated surfaces of an optical system without considerably increasing the computational complexity of the problem. This direct path to scaling the number of calculated surfaces is a result of the fact that the optical path length distance expressions do not depend on the number of considered surfaces.

Despite faster F-numbers and larger FOVs, freeform four-mirror designs bring in extra complexity to both mirror fabrication and system assembly compared to three-mirror designs. As a promising alternative, we have investigated a revived type of multi-reflection mirror systems (see Fig. 4), where at least one of the mirrors is passed twice in the ray path with desired overlap (not mutually centered to provide more flexible designs) [4].

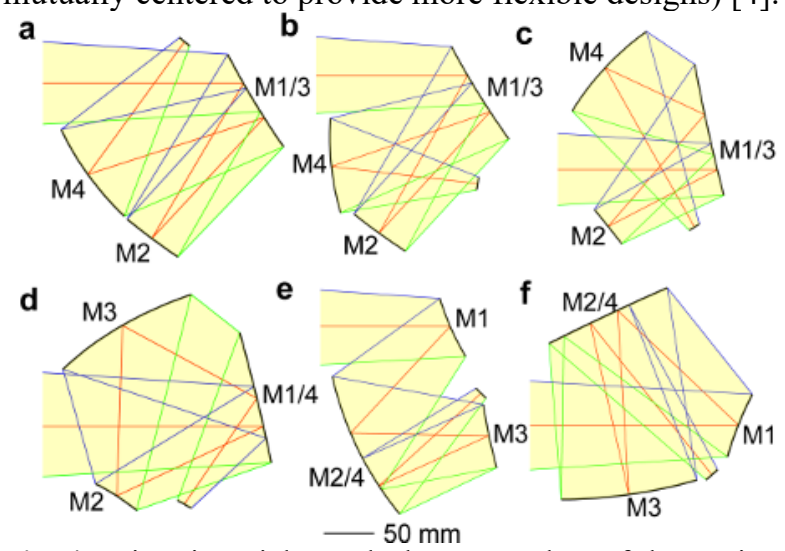

Fig. 4. "First time right" unlocks a new class of three-mirror, four-reflection systems with three different joint mirror options: a-c Joint M1\&M3. d Joint M1\&M4. e-f Joint M2/M4. 
This contrasts the well-known Offner designs where two individual mirrors share separate parts (no overlap) of a joint substrate without a desired overlap in their footprints. Regarding the four-reflection cases, there are three possibilities to generate the "double-pass surface", respectively mirrors M1/3, M1/4 and M2/4. Several showcases of each type have been calculated with the proposed "first time right" method, summarized in Fig. 4. Please note that all layouts are based on real raytracing with a focal length of $250 \mathrm{~mm}, \mathrm{~F} / 2.5$ and a full field of view of $7.2^{\circ} \times 7.2^{\circ}$. To conclude, the three-mirror, fourreflection concept can yield high-performing yet compact designs, opening a largely extended design space to explore and seek new competitive freeform systems [5].

\section{Discussion}

Equipping lens- and mirror-based optical systems with freeform optical surfaces makes it possible to deliver highly original imaging functionalities with superior performance. Compared to their traditional (a)spherical counterparts, freeform systems can offer an enhanced field-of-view, increased light-collection efficiencies, larger spectral band and higher compactness. Until now mathematical models and design strategies for freeform optics remained limited and failed to provide streamlined solutions. In particular, the identification of a suitable initial design has often proven to be a painstaking and time-consuming "trial-and-error" process.

In this article, we reviewed the first deterministic direct design method for freeform optical systems that is not restricted by the aberration terms that can be controlled and corrected. The method allows a highly systematic generation and evaluation of directly calculated freeform design solutions that can be readily used as excellent starting points for further optimization. As such, this new method allows the straightforward generation of "first time right" initial designs that enable a rigorous, extensive, and real-time evaluation in solution space when combined with available local or global optimization algorithms.

The authors acknowledge funding by VUB-OZR, BELSPOIAP, FWO [12C9817N], IOF, Hercules/FWO and the Methusalem foundations.

\section{References}

1. F. Duerr, H. Thienpont, Light Sci. Appl. 10, 95 (2021)

2. A. Bauer, E.M. Schiesser, J.P. Rolland, Nat. Commun. 9, 1756 (2018)

3. S.R. Kiontke, Proc. SPIE 9575, 95750G (2015)

4. D.R. Shafer, Appl. Opt. 16, 1178 (1977)

5. Y. Nie, D.R. Shafer, H. Ottevaere, H. Thienpont, F. Duerr, Opt. Express 29, 17227 (2021) 